\title{
Avaliação de livros de divulgação científica acerca da Mecânica Quântica
}

\author{
Fernanda Oliveira Simon \\ Renata Nascimento Nogueira \\ Flávio César Vicentin
}

\begin{abstract}
Resumo
Apesar da mecânica quântica já ser um tema oferecido em cursos universitários de engenharia, a forma como ela é apresentada envolve métodos matemáticos complexos, que dificultam o entendimento de alunos com pouca bagagem matemática. Este trabalho se propõe a analisar um conjunto de livros de divulgação científica que abordam o tema da mecânica quântica com vistas sua utilização em cursos de nível superior.
\end{abstract}

Palavras-chave: mecânica quântica, livros de divulgação científica, ensino superior.

\begin{abstract}
Although quantum mechanics has to be a subject offered in university engineering courses, how it is offered involves complex mathematical methods, which hinder the understanding of students with little math knowledge. This study aims to analyze a set of science books that address the topic of quantum mechanics with a view its use in undergraduate courses.
\end{abstract}

Keywords: quantum mechanics, science books, undergraduate courses.

\section{Introdução}

As aplicações da Física Moderna estão cada vez mais presentes no nosso cotidiano. Uma simples ida a um shopping center em que qualquer pessoa se depara com uma porta que abre e fecha de forma automática ou mesmo a utilização de células fotoelétricas, que transformam energia solar em elétrica para alimentar residências, são exemplos práticos da utilização da Física Moderna em nossas vidas. Apesar dessa realidade, muitos alunos do ensino médio ou mesmo de graduação desfrutam dessas tecnologias sem imaginar que elas nasceram de um árduo estudo e avanço da Física do Século XX.

No tocante ao Ensino de Física Moderna nos cursos de graduação no Brasil - em particular nos cursos de Engenharia e Computação -, as publicações nessa área ainda encontram-se incipientes se comparadas às desenvolvidas para o Ensino Médio.

Segundo Pantoja et al (2011), o número de pesquisas acerca do ensino de Mecânica Quântica (MQ) tem aumentado nos últimos anos, embora ainda seja considerado muito baixo. Em 
uma busca por vinte e um periódicos, entre os anos de 1999 e 2009, os autores encontraram apenas sessenta artigos sobre o tema, sendo que doze foram publicados em 2008 e 15 em 2009.

A maioria dos artigos encontrados por Pantoja et al (2011) foram enquadrados nas categorias "propostas didáticas" e "implementação de propostas didáticas". Poucos foram os artigos que se referiam à análise curricular, críticas aos cursos e concepções de estudantes e professores acerca da MQ. Neste último aspecto boa parte dos trabalhos está focada na construção de instrumentos para se avaliar estas concepções e há pouca discussão acerca das concepções em si.

Apesar disso, Perfoll e Junior (2006) apontam que já existe um número grande de Instituições que, por iniciativa própria, abordam a Física Moderna Contemporânea em seus currículos. Como não há uma legislação que evidencie esta necessidade, fica a cargo de cada instituição oferecê-la ou não (Lemes e Rezende Junior, 2011). Assim, o tempo destinado a elementos de Física Moderna Contemporânea precisa ser ampliado nas respectivas estruturas curriculares dos cursos de engenharia, tanto teórico como experimental.

Além disso, Greca et al (2001) salientam que a abordagem tradicional das disciplinas básicas de física para os cursos de ciências exatas ressaltam aspectos históricos, destacando mais as características clássicas do que as quânticas dos fenômenos. Quando recebem informações acerca da MQ estas são apresentadas na forma de equações complexas sem vínculo com os fenômenos em si. Desta forma, os cursos introdutórios não permitem que o aluno crie uma nova maneira de pensar sobre a realidade e cursos mais avançados (que exigem mais matemática) também não são capazes de discutir as concepções dos alunos acerca dos fenômenos quânticos.

Segundo Montenegro e Pessoa Jr. (2002), os cursos possuem ênfases excessivas em cálculos matemáticos, o que não permite aos alunos aprenderem os conceitos e fazerem considerações acerca das questões interpretativas. Desta forma surge a necessidade de se reavaliar os currículos dos cursos superiores de $\mathrm{MQ}$, especificamente no tocante a sua característica intensamente matemática.

Neste sentido, este artigo se propõe a avaliar um conjunto de livros de divulgação científica que abordam temas de $M Q$, com vistas a sua inclusão em disciplinas que trabalhem tópicos de MQ para alunos de engenharia de uma forma mais conceitual.

\section{O ensino de Mecânica Quântica no ensino superior}

Pesquisas realizadas por Perfoll e Rezende Jr (2006) acerca do ensino de Física Moderna Contemporânea para o ensino de Engenharia incluíram a análise das estruturas dos cursos de graduação em Engenharia de 177 Instituições de ensino superior, tanto privadas quanto públicas em todas as regiões do país, sendo que somente 54 destas disponibilizaram o ementário, 
resultando em 161 matrizes curriculares analisadas, de diversas áreas de Engenharia. Esse trabalho mostrou que já existe um número grande de Instituições que, por iniciativa própria, abordam a Física Moderna Contemporânea em seus currículos. Contudo, segundo esses autores, esses dados não indicam necessariamente que a Física do século XX já esteja inserida satisfatoriamente no ensino superior.

A MQ é introduzida nos cursos universitários de forma ainda fortemente relacionada com elementos da Física Clássica, considerando seus pressupostos mais básicos e complexos, seus fundamentos, além de toda a sua história, como a mecânica newtoniana. Essa abordagem tradicional, contudo, direciona para um segundo plano o fato de que os objetos de estudo na MQ são advindos de uma natureza diversa daqueles objetos clássicos (Guerra et al, 1998). Nesse sentido, muitos problemas filosóficos e de aprendizagem emergem ao pensar o ensino da $\mathrm{MQ}$ como conteúdo para o ensino superior (Greca et al, 2001). Ou seja, as abordagens típicas não criam condições para que os alunos aprendam os fenômenos e princípios da $M Q$, sendo esta matéria considerada "esotérica" e com poucas ligações com o mundo real.

Além disso, Rocha et al (2010), apontam que em vários cursos o ensino de MQ trabalha muito mais com a resolução de problemas envolvendo equações diferenciais e poucos aspectos relacionados aos conceitos fundamentais. Assim, colocam a necessidade de se criarem cursos complementares de curta duração para alunos de licenciatura e outras áreas (entre elas a engenharia) em que sejam abordados tópicos de MQ numa linguagem menos matemática, mas que sejam discutidos os conceitos fundamentais desta teoria. Os autores propõem que nestes "mini-cursos" sejam abordados os experimentos de fenda dupla, superposição de ondas, superposição de vetores no plano, estados quânticos e observáveis e espaços lineares. Além disso, consideram importante a abordagem dos primeiros postulados da MQ e suas implicações, como por exemplo, o emaranhado quântico, os relógios atômicos e a criptografia quântica.

Na opinião de Perfoll e Junior (2006) engenheiros competentes, atualizados e com amplo treinamento em pesquisa são a condição essencial do avanço da pesquisa industrial e da criação de novos produtos, de novos materiais e de serviços nas áreas tecnológicas. Para tanto, o ensino de graduação em Engenharia dever ser modernizado e atividades de pesquisa interdisciplinares devem ser induzidas e apoiadas. E com a presença cada vez mais marcante da Física Moderna Contemporânea em produtos que utilizamos em nosso cotidiano, são evidentes os interesses e investimentos do mercado nessas tecnologias, requerendo para tanto profissionais aptos a desenvolvê-las (Nguyen e Pudlowski, 2007).

No entanto, tradicionalmente, os cursos de graduação em engenharia apresentam a seguinte estrutura: "disciplinas básicas e disciplinas profissionais". As primeiras versam sobre ciências básicas (Física e Matemática principalmente), ao passo que a segunda é composta por um conjunto de disciplinas cujos conteúdos são específicos para cada modalidade de engenharia. Este núcleo é complementado por disciplinas eletivas de caráter mais geral (Maines, 2001). Todas estas 
disciplinas são encaradas de modo separado, não se relacionando, como se pertencessem a universos distintos.

Mesmo dentro de cada uma dessas estruturas, as disciplinas que as compõe também não se relacionam. As atividades de ensino apresentam-se compartimentadas, não sendo capazes de gerarem uma visão coerente de conjunto. Neste contexto, a principal característica da prática docente universitária tem sido a de apenas proporcionar um volume cada vez maior de informações aos estudantes (Ferreyra e González, 2000).

O problema é complexo e seria ingenuidade de nossa parte acreditar em soluções únicas. Pensamos que existe a necessidade de mudanças reais na estrutura das aulas, alterando os procedimentos de trabalhos que se desenvolvem em sala de aula (Almeida et al, 2004). A exposição do professor continua (e continuará) sendo essencial. Porém, os alunos precisam ser preparados para isso. Ou seja, tais exposições serão de grande utilidade se vierem a preencher uma lacuna no conhecimento dos alunos. A psicologia educacional nos explica que isso tem grande probabilidade de ocorrer quando, dada uma situação-problema, os alunos estão intrigados buscando um tipo de solução. Para isso, já propuseram algumas hipóteses e tentaram testá-las, mas mesmo assim não obtiveram soluções satisfatórias. Neste contexto, o professor deixa de ser um transmissor de informações para ser um orientador de "pesquisadores novatos" (Burns e Chisholm, 2005).

Para que essas ideias possam funcionar é preciso primeiro ter uma boa atividade, um bom problema que não seja de solução trivial, permita várias soluções e que os alunos sejam capazes de enfrentá-lo propondo e testando hipóteses. Para isso, as atitudes de professores e alunos diante do ensino também devem mudar. Talvez esta seja uma das grandes dificuldades, pois neste processo não caberá mais atitude passiva cultivada ao longo de vários anos na academia. Para a resolução de um problema mais próximo daqueles que serão encontrados nas indústrias é preciso ter atitudes pró-ativas frente à realidade.

A presença da MQ na vida prática e no imaginário deve ser ainda maior no futuro, à medida que novas tecnologias se desenvolvam calcadas em suas bases teóricas. Neste quadro, a obtenção de algum conhecimento, ainda que superficial, da descrição quântica da Natureza pode jogar um papel importante no exercício de diversas profissões e, por isso, começa a se tornar relevante numa formação profissional de boa qualidade.

Neste sentido, os livros de divulgação científica podem fornecer elementos para se discutir aspectos da $\mathrm{MQ}$ com uma abordagem de caráter mais qualitativo, que não exija uma formação muito profunda em matemática, mas ao mesmo tempo seja rigorosa na apresentação dos conceitos trabalhados. 


\section{Metodologia}

Primeiramente foi realizado um levantamento de livros de divulgação científica que apresentassem conceitos de Mecânica Quântica. A partir deste levantamento, foram selecionados dez livros para análise.

Um aspecto importante a ser atacado foi a seleção de textos que abordassem a Mecânica Quântica _de maneira qualitativa, sem envolver cálculos matemáticos mais sofisticados, de forma que pudessem ser utilizados por alunos da graduação a partir do primeiro semestre.

Uma vez selecionado o material, procedeu-se uma análise de conteúdo do texto. Nesta análise foram avaliados os seguintes aspectos: grau de adequação ao público-alvo, conceitos abordados, adoção de formulações matemáticas ou não e se faziam menção a experimentos ou não.

\section{Resultados}

A partir do levantamento de livros de divulgação sobre mecânica quântica disponíveis e que continuam sendo publicados, foi selecionado um conjunto de dez livros para serem analisados com vistas a sua potencial utilização em cursos de nível superior. Vale ressaltar que não foram analisados livros técnicos sobre o tema que envolvessem cálculos matemáticos mais avançados, dando ênfase na seleção dos livros para conceitos e abordagens fenomenológicas. Buscaram-se livros que abordassem o tema de uma forma mais qualitatival ou que apresentassem aplicações práticas.

O quadro abaixo mostra os livros analisados.

Quadro 1 - Livros analisados que abordam conceitos de mecânica quântica

\begin{tabular}{lll} 
Autor/ ano & Título do livro & Capítulos analisados \\
\hline Feynman (2005) & Física em 12 lições: fáceis e não tão fáceis & Capítulo 6 \\
Gamow (2010) & Biografía de la física & Capítulos 7 e 8 \\
Gleiser (1997) & A dança do universo & Capítulo 8 \\
Hawking (2001) & O universo numa casca de noz & Capítulo 2 \\
Hawking e Mlodinow & O Grande Projeto & Capítulos 3 e 5 \\
(2011) & & \\
Bohr (1995) & Física atômica e conhecimento humano & todos
\end{tabular}




\begin{tabular}{|c|c|c|}
\hline Autor/ ano & Título do livro & Capítulos analisados \\
\hline McEvoy e Zarate (2012) & $\begin{array}{l}\text { Entendendo teoria quântica: um guia } \\
\text { ilustrado }\end{array}$ & todos \\
\hline Polkinghorne (2002) & Teoria quântica & todos \\
\hline Pinto Neto (2010) & $\begin{array}{l}\text { Teoria e interpretações da mecânica } \\
\text { quântica }\end{array}$ & todos \\
\hline $\begin{array}{l}\text { Paula; Alves e Mateus } \\
\text { (2011) }\end{array}$ & $\begin{array}{l}\text { Quântica para iniciantes: investigações e } \\
\text { projetos }\end{array}$ & $\begin{array}{l}\text { Do capítulo } 4 \text { em } \\
\text { diante }\end{array}$ \\
\hline
\end{tabular}

O primeiro critério foi uma avaliação do grau de adequação ao público-alvo. Os livros foram analisados e divididos em três categorias: alto (livro de fácil e rápido entendimento, voltado para público leigo), médio (livros com formulações conceituais mais sofisticadas, mas que poderiam ser usados por alunos do ensino superior) e baixo (livros com formulações matemáticas que seriam mais adequados à pós-graduação).

O segundo critério foi fazer um levantamento dos conceitos abordados e, num terceiro momento, foi realizada uma avaliação sobre a adoção de fórmulas matemáticas ou não. Por fim, verificou-se se o livro apresentava experimentos sobre o tema, tanto experimentos mentais (do tipo "imagine uma bola que voa"....) como experimentos comuns que são realizados em laboratórios.

O quadro 2 mostra uma síntese dos resultados destas análises.

Quadro 2- Análise dos livros de divulgação.

\begin{tabular}{|c|c|c|c|c|}
\hline Livro & $\begin{array}{l}\text { Grau de } \\
\text { adequação }\end{array}$ & Conceitos abordados & $\begin{array}{l}\text { Uso de fórmulas } \\
\text { matemáticas }\end{array}$ & $\begin{array}{l}\text { Menção a } \\
\text { experimentos }\end{array}$ \\
\hline $\begin{array}{l}\text { Feynman } \\
(2005)\end{array}$ & Médio & $\begin{array}{l}\text { Princípio da incerteza } \\
\text { Formação dos átomos } \\
\text { Movimento dos elétrons } \\
\text { Eletrodinâmica quântica. }\end{array}$ & $\begin{array}{l}\text { Sim. Apenas apresenta } \\
\text { fórmulas sem entrar em } \\
\text { cálculos. }\end{array}$ & Não \\
\hline
\end{tabular}




\begin{tabular}{|c|c|c|c|c|}
\hline Livro & $\begin{array}{l}\text { Grau de } \\
\text { adequação }\end{array}$ & Conceitos abordados & $\begin{array}{l}\text { Uso de fórmulas } \\
\text { matemáticas }\end{array}$ & $\begin{array}{l}\text { Menção a } \\
\text { experimentos }\end{array}$ \\
\hline $\begin{array}{l}\text { Gamow } \\
(2010)\end{array}$ & $\begin{array}{l}\text { Médio. No } \\
\text { entanto o } \\
\text { livro está } \\
\text { escrito em } \\
\text { espanhol o } \\
\text { que pode } \\
\text { dificultar o } \\
\text { entendime } \\
\text { nto de } \\
\text { alguns } \\
\text { alunos. }\end{array}$ & $\begin{array}{l}\text { Estrutura do átomo } \\
\text { Experimento de Thomson } \\
\text { de medição da massa do } \\
\text { elétron } \\
\text { Descoberta dos raios-X } \\
\text { Modelos atômicos de } \\
\text { Rutherford e Bohr } \\
\text { Catástrofe do ultravioleta } \\
\text { Princípio da incerteza } \\
\text { Antimatéria. }\end{array}$ & $\begin{array}{l}\text { Sim. Apresenta fórmulas } \\
\text { e discorre sobre seus } \\
\text { significados e resultados } \\
\text { potenciais. }\end{array}$ & $\begin{array}{l}\text { Sim. Menciona } \\
\text { alguns } \\
\text { experimentos } \\
\text { mentais. }\end{array}$ \\
\hline $\begin{array}{l}\text { Gleiser } \\
(1997)\end{array}$ & Médio & $\begin{array}{l}\text { Cavidade de Kirchhoff } \\
\text { (corpo negro) } \\
\text { Mostra a história da } \\
\text { mecânica quântica } \\
\text { mencionando as } \\
\text { contribuições de Planck, } \\
\text { Einstein e Boltzman para o } \\
\text { desenvolvimento dos } \\
\text { conceitos da teoria } \\
\text { quântica, enfatizando os } \\
\text { dilemas e posicionamentos } \\
\text { de cada um. }\end{array}$ & Não & $\begin{array}{l}\text { Sim. Menciona } \\
\text { alguns } \\
\text { experimentos } \\
\text { mentais. }\end{array}$ \\
\hline $\begin{array}{l}\text { Hawking } \\
(2001)\end{array}$ & Médio & $\begin{array}{l}\text { Princípio da incerteza } \\
\text { Distribuição de } \\
\text { probabilidades } \\
\text { Efeito Casimir } \\
\text { Spin } \\
\text { Comportamento da } \\
\text { partícula } \\
\text { Estado fundamental }\end{array}$ & Não & $\begin{array}{l}\text { Sim. Alguns } \\
\text { experimentos } \\
\text { mentais. }\end{array}$ \\
\hline
\end{tabular}




\begin{tabular}{|c|c|c|c|c|}
\hline Livro & $\begin{array}{l}\text { Grau de } \\
\text { adequação }\end{array}$ & Conceitos abordados & $\begin{array}{l}\text { Uso de fórmulas } \\
\text { matemáticas }\end{array}$ & $\begin{array}{l}\text { Menção a } \\
\text { experimentos }\end{array}$ \\
\hline $\begin{array}{l}\text { Hawking e } \\
\text { Mlodinow } \\
(2011)\end{array}$ & Baixo & $\begin{array}{l}\text { Histórias alternativas da } \\
\text { MQ. Superposição quântica. } \\
\text { Experimento de dupla fenda } \\
\text { Princípio da incerteza } \\
\text { Trajetórias de Feynman. }\end{array}$ & Não & $\begin{array}{l}\text { Sim. Alguns } \\
\text { experimentos } \\
\text { mentais. }\end{array}$ \\
\hline Bohr (1995) & Médio & $\begin{array}{l}\text { Estrutura atômica } \\
\text { Quantum } \\
\text { Radiação de Planck } \\
\text { Debates epistemológicos } \\
\text { com Einstein. }\end{array}$ & Sim. Apenas citações. & Não. \\
\hline $\begin{array}{l}\text { McEvoy e } \\
\text { Zarate (2012) }\end{array}$ & Alto & $\begin{array}{l}\text { Anti-matéria } \\
\text { Estrutura atômica } \\
\text { (Heisenberg e Schrödinger) } \\
\text { Dualidade onda-pártícula } \\
\text { Efeito fotoelétrico } \\
\text { Eletrodinâmica } \\
\text { Equipartição de energia } \\
\text { Espectros de absorção e luz } \\
\text { de linha brilhante. } \\
\text { Conceito de fóton } \\
\text { Lei da radiação } \\
\text { Princípio da incerteza } \\
\text { Conceito de quantum }\end{array}$ & $\begin{array}{l}\text { Sim. No entanto, apenas } \\
\text { apresenta as equações } \\
\text { para ilustrar os } \\
\text { conceitos. Não faz } \\
\text { cálculos com elas. }\end{array}$ & Não \\
\hline
\end{tabular}




\begin{tabular}{|c|c|c|c|c|}
\hline Livro & $\begin{array}{l}\text { Grau de } \\
\text { adequação }\end{array}$ & Conceitos abordados & $\begin{array}{l}\text { Uso de fórmulas } \\
\text { matemáticas }\end{array}$ & $\begin{array}{l}\text { Menção a } \\
\text { experimentos }\end{array}$ \\
\hline $\begin{array}{l}\text { Polkinghorne } \\
\text { (2002) }\end{array}$ & Médio & $\begin{array}{l}\text { Natureza da luz } \\
\text { Efeito fotoétrico } \\
\text { Catástrofe ultravioleta } \\
\text { Estrutura atômica } \\
\text { Espalhamento de Compton } \\
\text { Fendas duplas e } \\
\text { sobreposição } \\
\text { Probabilidades e amplitudes } \\
\text { Princípio da incerteza } \\
\text { Lógica quântica } \\
\text { Tunelamento } \\
\text { Computação quântica } \\
\text { Teoria quântica de campos }\end{array}$ & $\begin{array}{l}\text { Sim. Ao final apresenta } \\
\text { um apêndice } \\
\text { matemático com as } \\
\text { principais fórmulas. No } \\
\text { entanto, não realiza } \\
\text { nenhum cálculo com } \\
\text { elas. }\end{array}$ & Não \\
\hline $\begin{array}{l}\text { Pinto Neto } \\
(2010)\end{array}$ & Médio & $\begin{array}{l}\text { Interpretação de } \\
\text { Copenhaguen } \\
\text { Teoria de Broglie-Bohm } \\
\text { Interpretação de vários } \\
\text { mundos } \\
\text { Teoria quântica e } \\
\text { cosmologia }\end{array}$ & $\begin{array}{l}\text { Sim. Apresenta as } \\
\text { fórmulas mais } \\
\text { sofisticadas e realiza } \\
\text { alguns cálculos. }\end{array}$ & Não \\
\hline
\end{tabular}




\begin{tabular}{|c|c|c|c|c|}
\hline Livro & $\begin{array}{l}\text { Grau de } \\
\text { adequação }\end{array}$ & Conceitos abordados & $\begin{array}{l}\text { Uso de fórmulas } \\
\text { matemáticas }\end{array}$ & $\begin{array}{l}\text { Menção a } \\
\text { experimentos }\end{array}$ \\
\hline $\begin{array}{l}\text { Paula; Alves } \\
\text { e Mateus } \\
\text { (2011) }\end{array}$ & Médio & $\begin{array}{l}\text { Espectro de radiação } \\
\text { luminosa } \\
\text { Fotoluminescência } \\
\text { Fosforescência } \\
\text { Fluorescência } \\
\text { Célula fotovoltaica } \\
\text { Estrutura atômica e da } \\
\text { matéria }\end{array}$ & Não & $\begin{array}{l}\text { Apresenta } \\
\text { somente } \\
\text { experimentos. }\end{array}$ \\
\hline & & Semicondutores & & \\
\hline
\end{tabular}

Analisando os dados apresentados no quadro 2, observou-se que apenas 1 dos livros (Hawking e Mlodinow, 2011) foi considerado com baixo grau de adequação ao público-alvo. Este livro, apesar de não apresentar formulações matemáticas, apresenta os conceitos de uma forma mais elaborada, que dificulta o entendimento para aqueles que não estejam familiarizados com o tema. Neste sentido, este não seria um material adequado para trabalhar os conceitos de mecânica quântica com os alunos de graduação em ciências exatas, mas poderia ser adotado como bibliografia complementar.

A maioria dos livros avaliados (8) foi considerada com um grau médio de adequação ao público-alvo, ou seja, são livros que apresentam os conceitos numa linguagem mais sofisticada. É exigida do leitor certa atenção no momento da leitura. Dentre estes, três não utilizam formulações matemáticas, três apenas citam algumas fórmulas ilustrativas e dois não apenas citam, mas apresentam também alguns cálculos e considerações acerca das fórmulas. No entanto, os cálculos apresentados não requerem pré-requisitos matemáticos sofisticados. No que se refere aos experimentos, a metade (quatro) apresenta alguns experimentos mentais do tipo "imagine .....", isto é, não são experimentos para serem realizados em laboratório, três não fazem alusão a nenhum experimento e um deles (Paula; Alves e Mateus, 2011) somente apresenta roteiros de experimentos.

Este último livro é muito interessante pois, além de apresentar os experimentos, inclusive com figuras ilustrativas com o passo a passo, também apresenta os conceitos-chave envolvidos, uma lista de material necessário e uma breve explicação dos resultados. Os experimentos envolvendo conceitos de mecânica quântica referem-se tanto a explicações de modelos como projetos e aplicações práticas. Além disso, em seus capítulos finais apresenta considerações sobre 
o uso do livro em sala de aula e oferece ideias de projetos para feiras de ciência, cultura e tecnologias. Desta forma, este livro configura-se mais como um manual para o professor para planejar os experimentos e não como um livro para o aluno.

Um dos livros analisados (McEvoy e Zarate, 2012) é realmente um livro voltado para o público leigo e com alto grau de adequação para alunos das séries iniciais da graduação. O livro apresenta conceitos sofisticados de forma simples e com leitura tranquila. As fórmulas matemáticas apresentadas são apenas ilustrativas. O livro também não faz menção a experimentos, logo, apesar de ser o que nos pareceu mais adequado a alunos com o perfil considerado, não deve ser a única bibliografia adotada, pois é importante o contato também com aspectos experimentais da teoria.

Assim, dos dez livros analisados apenas um (Hawking e Mlodinow, 2011) não seria adequado para uso em cursos de graduação. Os demais apresentam os conceitos de forma rigorosa, mas numa linguagem acessível o que torna o entendimento mais fácil.

\section{Considerações finais}

Frente aos grandes avanços tecnológicos nos últimos anos, com o surgimento de dispositivos e equipamentos que utilizam os conceitos de $\mathrm{MQ}$, um profissional com uma visão mais ampla sobre o tema, poderia incrementar o avanço do setor tecnológico e de inovação em nosso país, com crescimento, por exemplo, das patentes depositadas.

No entanto, o ensino de mecânica quântica em cursos de graduação na área de exatas, apesar de começar a ser implementado em diversas instituições de ensino superior, ainda possui uma estrutura voltada para aspectos matemáticos, em detrimento de discussões acerca dos conceitos fundamentais.

O que propomos aqui é uma maior ênfase em aspectos qualitativos e fenomenológicos, além de uma abordagem dos conceitos envolvidos em aplicações práticas e produtos. Mais do que saber efetuar os cálculos envolvidos, é fundamental que o engenheiro entenda de maneira clara os conceitos para que ele possa conceber e criar novos artefatos tecnológicos. Além disso, ao terem conhecimento sobre tema, o engenheiro será capaz de participar de discussões envolvendo profissionais de diversas áreas, permitindo uma interdisciplinaridade.

Desta forma, bons livros de divulgação científica, ao apresentarem os conceitos- de forma rigorosa, podem se tornar uma opção plausível para discussões em disciplinas de mecânica quântica no ensino superior. Dos dez livros analisados neste artigo nove se mostraram adequados para o uso nas condições apresentadas.

Apesar disso, a mera utilização dos livros analisados em sala de aula não fará com que o tema seja abordado de forma mais conceitual, mas pode ser um suporte para o professor. É 
necessário que a didática do professor seja revista e que ele tenha um profundo conhecimento sobre o tema, para que possa trabalhar os conceitos de forma clara e motivadora sem envolver cálculos matemáticos sofisticados.

\section{Referências}

ALMEIDA, Norton de; SIMON, Fernanda Oliveira; BARROS FILHO, Jomar. Ensino de engenharia mecânica: uma nova metodologia para o ensino do funcionamento de um aparelho a jato. In: WORLD CONGRESS ON ENGINEERING AND TECHNOLOGY EDUCATION-2004, 2004, Santos/SP. Engineerring education in the Changing Society. Council of Researches in Education and Sciences, 2004. v. 1, p. 1-4.

BOHR, N. Física atômica e conhecimento humano. Rio de Janeiro: Ed. Contraponto, 1995.

BURNS, G. R.; CHISHOLM, C. U. From graduate to professional engineer in a knowledge organisation: does the undergraduate curriculum provide the basic skills? Global Journal of Engineering Education, v. 9, n.1, p. 89-98, 2005.

FERREYRA, A., GONZÁLEZ, E. M. Reflexiones sobre la enseñanza de la fisica universitaria. Enseñanza de las Ciencias. V. 18, n. 2, p. 189-199, 2000.

FEYNMAN, R. P. Física em 12 lições: fáceis e não tão fáceis. Rio de Janeiro: Ed. Ediouro. 2005

GAMOW, G. Biografia de la fisica. Madri: Ed. Alianza, 2010.

GLEISER, M. A dança do universo. São Paulo: Ed. Companhia das Letras, 1997.

GRECA, I. M.; MOREIRA, M.A.; HERSCOVITZ, V. E. Uma proposta para o ensino de Mecânica Quântica. Revista Brasileira de Ensino de Física, v. 23, n. 4, p. 444-457, 2001.

GUERRA, A.; FREITAS, J.; REIS, J. C.; BRAGA, M. A. A interdisciplinariedade no ensino de ciências a partir de uma perspectiva histórico-filosófica. Caderno Catarinense de Ensino de Física, v. 15, n. 1, p. 32-46, 1998.

HAWKING, S. O universo numa casca de noz. Rio de Janeiro: Ed. Nova Fronteira, 2001. HAWKING, S. e MLODINOW, L. Um nova história do tempo. Rio de Janeiro: Ed. Ediouro Sinergia, 2011.

MAINES, A. Ensino de engenharia - tendências e mudanças. In: CONGRESSO BRASILEIRO DE ENSINO DE ENGENHARIA, 29., 2001, Porto Alegre. Anais eletrônico. Porto Alegre: ABENGE, 2001, p. FCU -45 a FCU - 50 [CD-ROM].

McEVOY, J.P.; ZARATE, O. Entendendo Teoria Quântica. São Paulo, Leya, 2012.

MONTENEGRO, R. L. ; PESSOA Jr., O. . Interpretações da Teoria Quântica e as Concepções dos Alunos de Física. Investigações em Ensino de Ciências (UFRGS), Porto Alegre, v. 7, n.2, 2002.

R. B. E. C. T., vol 7, núm. 2, mai-ago.2014 ISSN - 1982-873X 
NGUYEN, D. Q.; PUDLOWSKI, Z. J. Issues and challenges in engineering education and the future outlook of the engineering profession in Australia. Global Journal of Engineering Education, v. 11, n.2, p. 107-116, 2007.

PANTOJA, G. C. F.; MOREIRA, M. A.; HERSCOVITZ, V. E. Uma revisão da literatura sobre a pesquisa em ensino de Mecânica Quântica no período de 1999 a 2009. Revista Brasileira de Ensino de Ciência e Tecnologia, 4(3), P.1-34, 2011.

PAULA, H. F., ALVES, E. G. e MATEUS, A. L. Quântica para iniciantes: investigações e projetos. Belo Horizonte: Editora UFMG, 2011

PERFOLL, A. P.; REZENDE Jr., M. F. A física moderna e contemporânea e o ensino de engenharia: contexto e perspectivas. In: Congresso Brasileiro de Ensino de Engenharia, Passo Fundo, 2006. Disponível em: http://www.dee.ufma.br/ fsouza/anais/arquivos/11_97_581.pdf. Acesso em 01 out 2012.

PINTO NETO, N. Teorias e interpretações da mecânica quântica. São Paulo, Editora Livraria da Física; Rio de Janeiro, Centro Brasileiro de Pesquisas Físicas, 2010.

POLKINGHORNE, J. Teoria quântica. Poro Alegre, RS, L\&PM, Coleção L\&PM Pocket, vol 985, 2011.

ROCHA, C. R.; MOREIRA, M. A.; HERSCOVITZ, V. E. Introdução à Mecânica Quântica: uma proposta de minicurso para o ensino de conceitos e postulados fundamentais. Revista Brasileira de Ensino de Ciência e Tecnologia, v. 3, n. 1, 2010.

Renata Nascimento Nogueira: Possui graduação em Física (bacharelado) pela Universidade de São Paulo (1992), mestrado em Física pela Universidade de São Paulo (1995) e doutorado em Física pela Universidade de São Paulo (1999). Atualmente participa de um grupo de pesquisa da Anhanguera Educacional, com bolsa da Funadesp. Tem vínculo como horista na Anhanguera Educacional, na Escola Antonietta e Leon Feffer e na Universidade Paulista. Tem experiência na área de Física, com ênfase em Física da Matéria Condensada e em Ensino de Física.

Flávio César Vicentin: Possui graduação em Física pela Universidade Estadual de Campinas (1986), mestrado (1990) e doutorado (1999) em Física pela Universidade Estadual de Campinas. Exerce a função de físico desde 1992 no Laboratório Nacional de Luz Síncrotron,Campinas/SP., e desde 2011 exerce o cargo de pesquisador e coordenador da linha de luz de raios-X moles (SXS). É professor titular, em tempo parcial, da Anhanguera Educacional na Faculdade Comunitária de Campinas, unidades 1 e 2, em Campinas/SP desde 2004. Foi professor visitante do departamento de Física da Universidade Federal de Sergipe em 2002. Desenvolveu estágio científico em espectroscopia de absorção de raios-X usando luz síncrotron no LURE(Laboratoire pour I'Utilisation du Rayonnement Électromagnétique) localizado na Universidade Paris-Sud - Orsay - 
França. Tem experiência na área de Física, com ênfase em espectroscopia de raios-X em sistemas biológicos e do meio ambiente, atuando principalmente nos seguintes temas: instrumentação e ótica de raios-X (espelhos, monocromadores, estações experimentais, etc.), radiação síncrotron, propriedades eletrônicas e magnéticas de materiais, espectroscopia de absorção e fotoemissão de raios- $X$ moles. 